\title{
Abstracts of Scientific Presentations at the 2018 National Annual Conference of the Critical Care Society of Southern Africa
}

\section{3 - 26 August 2018, Durban Convention Centre, Durban}

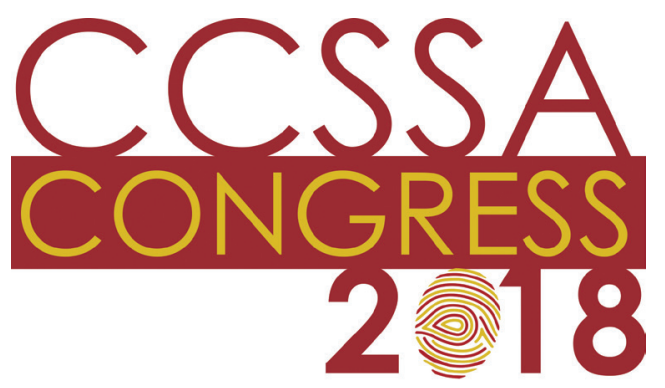

\section{Oral presentations}

\section{An analysis of referrals to a level-3 intensive care unit in a resource- limited setting in South Africa U V Jaganath, ${ }^{\star}$ K de Vasconcellos, D L Skinner, D Gopalan \\ Department of Anaesthesiology and Critical Care, University of KwaZulu-Natal \\ *ushirjaganath@gmail.com}

Background. With a shortage of intensive care unit (ICU) beds and rising healthcare costs, clinicians need to appropriately triage patient admissions into such units to avoid wasteful expenditure and unnecessary bed utilisation.

Objectives. To assess the nature, appropriateness and outcomes of referrals to a tertiary centre ICU.

Methods. A retrospective review of 500 ICU consults was performed. Data collected include patient and referring doctor demographics, diagnosis and comorbidities and biochemical and haemodynamic parameters of the patient, all of which then being cross-referenced to the outcome of the ICU consultation.

Results. Junior medical officers made up 164 (32.8\%) of the consultations. Although specialist supervision was available in 459 cases, they were only utilised for 339 (73.9\%) cases. Of the consultations, 102 (20.4\%) and $7(1.4 \%)$ took place at district hospitals and clinics, respectively. In $81(16.2 \%)$ consultations, the appropriate referral pathway was not followed. A total of 85 (17\%) patients were deemed futile admissions, with 25 (29.4\%) demising within 24 hours of consultation. Of the 204 accepted patients, 21 (10.3\%) demised before arrival. Of the 120 withdrawn consultations, $30(25 \%)$ patients demised during the consultation period, while $55(45.8 \%)$ did not require intensive care. Patients had an average of 2.5 dysfunctional organ systems and the respiratory system was most commonly affected.

Conclusion. More emphasis needs to be placed on the training of doctors to appropriately manage critically ill patients to prevent unnecessary referral and wastage of already scarce ICU beds.
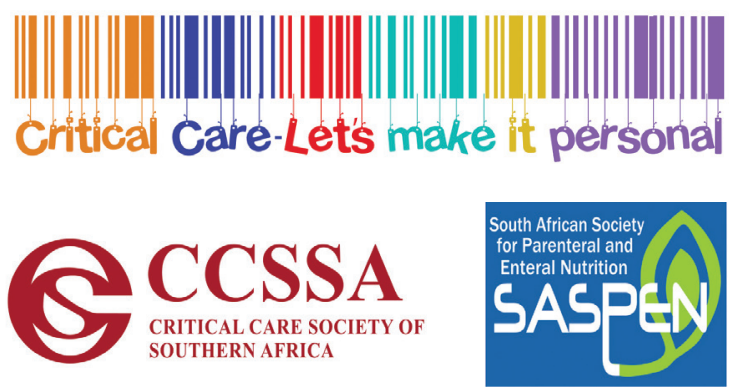

Opinions of doctors working in South African critical care units regarding unconsented testing and empirical treatment of HIV-positive patients in intensive care units

A K Asghar ${ }^{1 \star}{ }^{\text {R Wise },}{ }^{2}$ A M Carpenter, ${ }^{2}$ J N Mudali ${ }^{3}$

${ }^{1}$ Bethesda Hospital, Ubombo

${ }^{2}$ Edendale Hospital, Pietermaritzburg

${ }^{3}$ Department of Anaesthetics and Critical Care,University of KwaZulu-

Natal

*adam.asghar@gmail.com

Background. There is no consensus about acute management of HIV in the critical care setting. There exists a limited number of studies which demonstrate benefit of initiating antiretroviral therapy (ART) in intensive care units (ICUs). However, there are physiological, practical and ethical arguments against making this general practice.

Objectives. To describe the opinions of South African doctors working in the critical care setting about acute management of HIV in ICU.

Methods. A non-experimental, multi-centre, prospective, observational study was conducted with data collected using a questionnaire distributed to delegates of the Critical Care Society of South Africa (CCSSA) 2016 congress, and to members of the society's mailing list.

Results. There were 101 respondents, of whom $8 \%$ would not consider testing a critically ill patient for HIV (status previously unknown), as this would be unethical. Sixty-nine percent would consider commencing ART in a patient newly diagnosed with HIV during an inter-current critical illness. The factor most likely to guide them towards this was severity of illness. Concerns about ART toxicity, and lack of pre-ART initiation counselling, and potentially defaulting from therapy were the greatest concerns held by those clinicians who would not consider ART initiation.

Conclusion. Although the majority of clinicians support acute ART initiation in critically ill patients, there remain barriers to making this routine practice. Guidelines are thus needed to help clinicians navigate through the complex practical and ethical considerations in such cases. 
Intravenous artesunate for the

\section{treatment of severe malaria in the intensive care unit}

\section{R Tadzimirwa, S Omar, ${ }^{\star}$ J M Brown, I S Kalla \\ Department of Medicine, Department of Critical Care, Department of \\ Pulmonology, University of the Witwatersrand \\ *shahedicu@gmail.com}

Background. In South Africa, malaria is endemic in Mpumalanga, KwaZulu-Natal and Limpopo. During 2011 it was responsible for 87 deaths. The AQUAMAT and SEAQUAMAT trials showed superiority of intravenous (IV) artesunate over quinine. Artesunate (IV) remains unregistered in SA.

Objectives. To determine the observed mortality of patients with severe malaria requiring ICU and treated with IV artesunate.

Methods. We performed a retrospective chart review (April 2010 - April 2014) at two academic intensive care units (ICUs) in Johannesburg.

Results. In total, 56 patients were included. The mean (standard deviation (SD)) age of patients was 40.3 (11.6) years. There were 40 male patients (71.4\%). The mean (SD) Apache II score was 19 (5.4), with a predicted mortality of $32.2 \%$; $48 \%$ had at least one co-morbidity, with HIV being the most prevalent (32\%). More than two-thirds (71.4\%) of the patients had a travel history to a malaria-endemic area, $26.8 \%$ had no travel history and $1.8 \%$ unknown travel. We observed a lower-than-predicted mortality rate of $21.4 \%$ (standardised mortality ratio 0.67 ). Hazard ratio, relative risk and Glasgow Coma Scale (GCS) all improved significantly from admission to discharge $(p \leq 0.01)$. Markers of severe malaria, including acidaemia, bilirubin, urea and bleeding risk (platelets) also improved $(p \leq 0.01)$. Non-survivors had a lower GCS and worse acidaemia on D1 $(p \leq 0.01)$. Mechanical ventilation was associated with an increased risk of death, (odds ratio (OR) 35 (95\% confidence interval (CI) 7.0 - 182), as was vasopressor use (OR 4.3; 95\% CI 1.7 - 11.4).

Conclusion. Artesunate (IV) was found to be efficacious and associated with a lower-than-predicted mortality in patients with severe malaria requiring ICU admission.

\section{Tracheal tube cuff pressure monitoring: Assessing current practice in critically ill patients at Chris Hani Baragwanath Academic Hospital}

\section{A B Khan ${ }^{1+}$ S Omar, ${ }^{1}$ K Thandrayen ${ }^{2}$}

${ }^{1}$ Department of Critical Care, University of the Witwatersrand

${ }^{2}$ Department of Paediatrics, University of the Witwatersrand

*abatiya@gmail.com

Background. Intubated patients with a high tracheal tube cuff pressure (CP) are at risk of developing tracheal or subglottic stenosis. Recently, an increasing number of patients have presented to our hospital with these complications.

Objectives. To determine the frequency of tracheal tube cuff pressure measurements $(\mathrm{CPM})$, the range of $\mathrm{CP}$ and explore nursing knowledge regarding $\mathrm{CP}$ monitoring.

Methods. Frequency of CPM was assessed using a prospective intensive care unit chart review. An interventional component of CPM was performed next. Finally, a self-administered questionnaire was completed by nurses.

Results. Chart reviews and CPM were completed on 304 charts for 61 patients. Patients' ages ranged from 1 - 71 years, with a male preponderance (1.5:1). Eighty-seven percent of the charts did not have a documented CPM and only 12 charts had at least one measurement per shift. Only $17 \%$ of CPs were within the recommended range, with $59 \%$ being low.

The questionnaire was administered to 75 professional nurses, with a response rate of $51 \%$. Intensive care nursing experience ranged from 3 to 35 years, with $92 \%$ of respondents. Seventy-two percent of respondents reported measuring $\mathrm{CP}$ at least once per shift and knew the recommended cuff pressure range. Almost all respondents (94\%) knew of at least one complication of abnormal CP.

Conclusion. Current practice requires urgent revision. Development of a nursing guideline together with in-service training may improve compliance with CPM, and potentially decrease complications that arise from abnormal cuff pressure.

\section{An evaluation of current feeding practices and determination of barriers to adequate nutritional support in a multidisciplinary South African intensive care unit \\ E O B Elmezoughi, ${ }^{*} \mathrm{~K}$ de Vasconcellos}

Department of Anaesthesiology and Critical Care, University of

Kwazulu-Natal

*beladalandalus@yahoo.com

Background. Adequate nutritional support is crucial to optimising intensive care unit (ICU) outcomes.

Objectives. To assess adherence to current nutritional guidelines in critically ill patients in South Africa (SA). To identify risk factors for nonadherence to guidelines.

Methods. Retrospective observational chart review of nutritional practices during the first week of ICU admission in adult patients admitted to a tertiary ICU in Durban, SA, for $>48$ hours. The study was conducted from 1 December 2017 to 31 May 2018. The study received full ethical and institutional approval prior to commencing.

Results. The study included 150 patients, with a median age of 39 years and an ICU mortality rate of $28 \%$. Surgical patients accounted for $51 \%$ of admissions. Median admission SOFA score was 7, with $74.7 \%$ of patients requiring inotropic support. Enteral nutrition (EN) was initiated in $48 \%$ of patients within 48 hours of admission. Goal feeds were reached in $23 \%$ of patients by discharge, death, or day 7. Parenteral nutrition (PN) was initiated in $17 \%$ of patients. Patients with infectious aetiologies were more likely to receive EN, as were patients who underwent surgery during their ICU stay. PN was more common in postoperative patients and patients with shock. Common reasons for failure to initiate or increase EN included pending surgery or extubation, haemodynamic instability and failure to adhere to the feeding protocol for unspecified reasons.

Conclusion. Adequate nutrition is associated with reduced morbidity, ICU length of stay, mortality and improved functional outcomes. Avoiding barriers to adequate ICU nutrition and enhanced adherence to feeding protocols should be encouraged.

\section{Red blood cell concentrate transfusion strategies utilised at a tertiary-level paediatric intensive care unit: A descriptive study on impact and cost \\ P Radebe ${ }^{*} \mathbf{P}$ M Jeena}

Department of Paediatrics and Child Health, University of Kwazulu-Natal palessa.radebe@gmail.com

Background. The optimal haemoglobin threshold for red blood cell 
(RBC) transfusions in critically ill children with anaemia remains uncertain.

Objectives. To describe the RBC transfusion strategies, outcomes and costs for children with anaemia who were admitted to the paediatric intensive care unit (PICU) at Inkosi Albert Luthuli Central Hospital in Durban, South Africa.

Method. A retrospective analytical study was conducted at a single, tertiary PICU during 2015. RBC transfusions were classified as restrictive, modified liberal or mixed (multiple transfusion) strategies. The modified liberal group was sub-divided into a haemodynamically stable or unstable clusters. Transfusion-related effects, co-morbidities and mortality were described. The costs of transfused RBC concentrates and the associated levies were evaluated.

Results. A total of 118 of 556 admissions were enrolled in the study: 27 cases $(22.9 \%)$ were in the restrictive strategy; 68 cases $(57.6 \%)$ in the modified liberal strategy (haemodynamically stable 21 (30.9\%) and unstable 47 (69.1\%) cases); and 23 cases (19.5\%) in the mixed strategy. The study participants received 183 RBC transfusions. A trend towards increased mortality among the modified liberal v. the restrictive group $(27.9 \%$ v. $11.1 \% ; p=0.09)$ was observed. No differences were observed in the duration of intermittent positive-pressure ventilation, ICU stay and post-transfusion effects between the restrictive and modified liberal (haemodynamically stable and unstable) transfusion strategies. An expenditure of ZAR29 000 for avoidable levies was noted. A restrictive $\mathrm{RBC}$ concentrate transfusion strategy for patients in the modified liberal haemodynamically stable group would realise a saving of ZAR150 000 . Conclusion. The adoption of a restrictive day-time strategy for RBC transfusions at a tertiary PICU would be associated with a cost saving without a risk of increased mortality and morbidity.

\section{The effect of non-pharmacological interventions on the severity and duration of hypoactive delirium and delirium in postoperative cardio- thoracic surgery patients}

\section{A Kruger, ${ }^{\star}$ I M Coetzee, Z White}

Department of Nursing Science, University of Pretoria

arietakruger@gmail.com.

Background. The prevalence of hypoactive delirium and delirium in intensive care units (ICUs) can be as high as $80 \%$, and is characterised by decreased cognitive function, inattentive thinking, fluctuation of consciousness, disorientation and confusion, which could result in an increase of 6 months' mortality and cognitive impairment. If no screening tool for detection for hypoactive delirium and delirium is utilised, it will be undetected and the outcome will be worse if no non-pharmacological interventions are in place.

Objective. To assess the effect of non-pharmacological interventions compared to standard nursing care on the severity and duration of hypoactive delirium and delirium in ICU patients following cardio-thoracic surgery.

Method. A quasi-experimental, non-equivalent control group design was conducted among conveniently sampled postoperative cardiothoracic surgery participants $(N=30)$. The setting was a private hospital with 138 beds and 18 ICU beds based in Gauteng. Participants were screened daily for hypoactive delirium or delirium at $08 \mathrm{~h} 00$ and $16 \mathrm{~h} 00$ utilising the ICU delirium screen checklist (ICDSC) until no delirium. The difference in the ICDSC checklist scores was utilised for data analysis using descriptive statistics.
Results. The duration in hours from hypoactive delirium and delirium to no delirium in the intervention group (62.4 hours to no delirium) was significantly shorter than in the control group (72.3 hours to no delirium) ( $p=0.02$ ). ICU-bed cost estimation was R12 800/day.

Conclusion. Results support the effect of non-pharmacological interventions on the improvement of delirium in postoperative cardiothoracic patients.

Limitations. One ICU unit in a private hospital was used.

\section{Neonatal and paediatric parenteral nutrition prescription practices in South Africa \\ C S Flint, ${ }^{\star}$ E Van Niekerk, R Blaauw \\ Division of Human Nutrition, Stellenbosch University, Cape Town, South Africa \\ *cflintmasters@gmail.com}

Background. Parenteral nutrition (PN) is utilised to feed neonatal and paediatric patients in South African (SA) hospitals, but little is known regarding what influences and guides this clinical practice.

Objectives. To describe the current PN prescription practices and knowledge of prescribers in SA, and to establish factors that influence usage and adherence to the available guidelines.

Methods. A descriptive cross-sectional survey was conducted through an online questionnaire. PN prescriptions and knowledge of the ESPGHAN guidelines were assessed. Stratification of respondents was according to work sector (state/private) or professional group (dietitian/paediatrician). Results. A total of 72 respondents (58\% dietitians and $42 \%$ doctors; $47 \%$ private and $53 \%$ state sector) were included. Primary indications for PN use were gut intolerances and prematurity. Dietitians were more likely to calculate the protein requirements $(p<0.001)$, whereas doctors prioritised fluids. More than one-third (36\%) of prescribers commenced PN within 24 hours, and 67\% introduced lipid emulsions (IVLE) from PN initiation. The main reason for IVLE delay was habit. Infectious complications and financial constraints were the main reason for delaying or not using PN. Mean (standard deviation (SD)) practice score was $75 \%$ (17). The dietitians scored higher compared with doctors ( $82 \%$ (12) v. $65 \%(19) ; p<0.001)$. The mean knowledge score $(n=64)$ was $74 \%$ (12), with the dietitians scoring higher than doctors (77\% (13) v. $71 \%(11) \%) ;(p=0.04)$.

Conclusion. PN prescribing practices in SA have not been optimised for neonatal and paediatric patients. A multidisciplinary approach to PN feeding is paramount. Our findings emphasise the role of the dietitian in optimal feeding.

\section{Poster presentations}

\section{Respiratory distress syndrome following a biphasic anaphylactic reaction to morphine: A case report and review of the literature $\underline{\text { K U Tobi }},{ }^{1 *}$ G Kirenga, ${ }^{1}$ S Muhmuza, ${ }^{2}$ P Ruhato ${ }^{2}$ \\ ${ }^{1}$ Department of Surgery and Anaesthesia, University of Namibia ${ }^{2}$ Department of Anaesthesia, Critical Care and Emergency Medicine, University of Kigali \\ *tobikingsley265@gmail.com}

Background. A biphasic anaphylactic reaction is a very rare variant 
of the usual and more commonly seen monophasic anaphylactic reaction. However, recently it has been observed that biphasic anaphylactic reactions may not be as uncommon as previously believed. Furthermore, serious and life-threatening complications such as acute respiratory distress syndrome (ARDS) may ensue that require prompt intervention.

Case Report. We present a 16-year-old boy who was scheduled for bilateral orchidopexy under spinal anaesthesia. Anaesthesia was supplemented with intravenous $5 \mathrm{mg}$ midazolam, $50 \mathrm{mg}$ ketamine and $5 \mathrm{mg}$ morphine. Approximately 10 minutes after the administration of morphine, the patient developed an urticaria with a mucocutanoeus rash, which was promptly treated with $100 \mathrm{mg}$ intravenous hydrocortisone. The patient was transferred to the ward after an uneventful surgery and anaesthesia. However, after $\sim 6$ hours postoperatively, he developed shock, with a blood pressure reading of $80 / 40 \mathrm{mmHg}$, a heart rate of 40 beats per min and an $\mathrm{SpO}_{2}$ of $20 \%$. He was immediately resuscitated with endotracheal intubation, chest compression and intravenous adrenaline, and admitted to the ICU. He was managed in the ICU with a ventilatory and inotropic support, and discharged to the ward after 12 days.

Conclusion. Biphasic anaphylactic reaction to opioids, especially morphine, administered in the perioperative period, is not an unlikely event. One of the common consequences is ARDS, which would require ICU admission and ventilatory support. Awareness of this possibility, increased vigilance and prompt intervention are the sine qua non for preventing unfavourable outcomes.

\section{Changes in selected electrolytes in adult intensive care patients at the University Teaching Hospital (UTH), Lusaka, Zambia \\ N Sheyo \\ Department of Anaesthesia and Intensive Care, University of Zambia ninza.sheyo@gmail.com}

Background. The importance of regulating electrolyte levels is well recognized in most intensive care units. The extent of sodium and potassium imbalances in patients admitted to the Main Intensive Care Unit (MICU) at the University Teaching Hospital, Lusaka, Zambia, is unknown. Objective. This study aimed to clarify whether the abnormalities were associated with negative outcomes.

Methods. We conducted an observational cross-sectional study of patients admitted to the MICU. Blood samples were collected at 0 hours and 24 hours post admission. Normal serum concentrations of sodium and potassium were considered as $135-145$ and $3.5-4.5 \mathrm{mmol} / \mathrm{L}$, respectively. Values at 0 and 24 hours were compared with the Wilcoxon signed-rank test. Logistic regression analysis was used to investigate the relationship between electrolytes and mortality. A $p$-value $<0.05$ was considered statistically significant.

Results. 100 patients were included in this study with a mean (standard deviation (SD)) age of 36.8 (12.1) years. The mean (SD) sodium levels were $136.7(8.9) \mathrm{mmol} / \mathrm{L}$ and $139.0(11.6) \mathrm{mmol} / \mathrm{L}$, on admission and 24 hours post admission, respectively $(p=0.0051)$. Hypernatraemia was shown to be associated with an increased risk of death $(p=0.021)$ in the MICU, with an odds ratio of 4.0 (95\% confidence interval $1.2-12.9)$. Hyponatraemia was the most prevalent electrolyte imbalance.

Conclusion. There is a significant change in serum sodium levels 24 hours post admission. Hyponatraemia being the most prevalent.
Hypernatremia is significantly associated with mortality. Early correction of electrolyte imbalances in ICU can reduce mortality.

\section{The association between diaphragm contractile activity and maximal inspiratory pressure in critically ill patients}

\author{
L E Brouwer, ${ }^{1 *}$ A Lupton-Smith, ${ }^{1}$ C F N Koegelenberg, ${ }^{2}$ \\ S D Hanekom ${ }^{1}$ \\ ${ }^{1}$ Department of Health and Rehabilitation Sciences, Stellenbosch \\ University \\ ${ }^{2}$ Department of Medicine, Stellenbosch University \\ lindiegerber15@gmail.com
}

Background. Ventilator-induced diaphragmatic dysfunction (VIDD) causes a loss of the force-generating capacity of the diaphragm, and may contribute to weaning failure. Maximal inspiratory pressure (MIP) is used to measure inspiratory muscle strength. We investigated whether there is an association between diaphragm contractile activity and MIP. Objective. To determine whether the change in the contractile activity of the diaphragm during the course of mechanical ventilation was associated with post-extubation inspiratory muscle strength.

Method. Diaphragm thickness was measured daily with ultrasound in the zone of apposition using a $5-12 \mathrm{MHz}$ ultrasound transducer. Diaphragm thickness was measured at end-inspiration and endexpiration for three breaths. The diaphragm thickening fraction (DTF), representing the contractile activity, was calculated as the percentage change between thickness at end-inspiration and endexpiration. End-expiratory diaphragm thickness was measured and reported. MIP was measured within 24 hours of extubation.

Results. A total of 68 study participants (57\% male), with a mean (standard deviation) age of 45 (16) years, were studied. The median (range) duration of mechanical ventilation was 4 (1 - 29) days. There were no correlations between the rate of change in DTF while intubated $(r=-0.03 ; p=0.862)$, baseline $(r=0.17 ; p=0.22)$, last $(r=-0.04$; $p=0.85)$ or post-extubation DTF $(r=-0.02 ; p=0.87)$ and MIP. Endexpiratory thickness was not correlated with MIP at any time point.

Conclusion. Diaphragm contractile activity is not correlated with volitional measures of inspiratory muscle strength and may not be a suitable surrogate measure of respiratory muscle strength in this population of critically ill patients.

\section{A retrospective comparison of the burden of organophosphate poisoning in an intensive care unit in Soweto over two separate periods}

\section{K M San Pedro, L Torien, L R Mathivha, S Omar}

Department of Critical Care, University of the Witwatersrand *shahedicu@gmail.com

Background. Organophosphate poisoning (OPP) is a major healthcare burden, with 3 million cases reported annually, worldwide. The prognosis depends on the development of complications. Recent experience suggests an increase in complications secondary to OPP. The laboratory provided Aldicarb as the culprit 'rat poison' toxin in 2012. The manufacturer has since removed the product from the market. We analysed a currently available 'rat poison' in the informal market and identified Terbufos (organophosphate).

Objectives. To describe the differences among patients who were 
admitted to the intensive care unit (ICU) with a cholinergic toxidrome over two time periods: 2012 and 2017.

Methods. A retrospective review of the adult database at Chris Hani Baragwanath Academic Hospital was performed.

Results. A total of 41 patients were included: 9 (22\%) during 2012 and $32(78 \%)$ during 2017. Males constituted 54\% $(n=22)$. The median age was 26.5 years. All presented with cholinergic symptoms, and 95\% were intubated. Number of antidote days, ventilator days and ICU length of stay were longer in 2017 ( $p \leq 0.004)$. No patients required reintubation, tracheostomy or vasopressor support in 2012, while these were required in $25 \%, 23 \%$ and $13 \%$ of cases respectively, in 2017 . The odds ratio (OR) for ICU admission in 2017 was higher than in 2012 (OR 5.6 (95\% confidence interval 1.2 - 26).

Conclusion. There has been an increase in ICU admission for patients presenting with a cholinergic toxidrome in 2017 v. 2012. A shift from a market-regulated carbamate to an unregulated organophosphate may be responsible and is of important public health concern.

\section{The relationship between organisational culture, transformational leadership and organisational change outcomes in public intensive care units}

N Befile, $P$ J Jordan, ${ }^{\star}$ D Venter

School of Clinical Care Sciences, Nelson Mandela University portia.jordan@mandela.ac.za
Background. An organisational culture refers to those values and norms within an organisation that are prescribed to both employer and employees regarding how to behave. However, organisational culture should not be viewed in isolation, as culture and leadership are intertwined. Transformational leadership within the organisational culture serves to achieve goals, missions and aims by influencing, motivating and creating a mutual relationship between employer and employees, which brings about effective organisational change. The alignment of organisational culture and leadership with a hospital vision is important to ensure optimal healthcare delivery and organisational change.

Objectives. To explore whether a supportive organisational culture, transformational leadership and organisational change outcomes were prevalent in public intensive care units (ICUs).

Methods. The study followed a positivistic, quantitative paradigm. Non-probability sampling was used, which allowed the distribution of questionnaires to enrolled and registered nurses who were employed in public ICUs within Nelson Mandela Bay. We used a structured questionnaire that was validated in a previous study, and proved to be reliable as a Cronbach's alpha score of 0.80 . Descriptive and inferential statistics were used to analyse data.

Results. The results suggested a significant relationship between organisational culture, transformational leadership and organisational change outcomes in ICUs within Nelson Mandela Bay.

Conclusion. Nursing managers should adopt the transformational leadership style in order to ensure a therapeutic environment within the organisational culture, to enhance positive organisational change outcomes. 